\title{
Links among emotional awareness, somatic awareness and autonomic homeostatic processing
}

\author{
Kenji Kanbara* (1) and Mikihiko Fukunaga
}

\begin{abstract}
Emotional awareness and somatic interoceptive awareness are essential processes for human psychosomatic health. A typical trait of lacking emotional awareness related to psychosomatic symptoms is alexithymia. In contrast, alexisomia refers to the trait of lacking somatic awareness. Links between emotional and somatic awareness and homeostatic processing are also significant for the psychosomatic health. The purpose of the present paper is to review the links among emotional awareness, somatic interoceptive awareness and autonomic homeostatic processing. On the basis of the collected evidence, the following arguments were presented': (1) The main subcortical neural substrates for these processes are limbic-related systems, which are also responsible for autonomic functions for optimization of homeostatic efficiency. (2) Considerable studies have shown that autonomic activity and/or reactivity to stress correlate with both emotional and interoceptive awareness. A hypothesis was advocated about the links between the two types of awareness and autonomic function: Autonomic dysfunction, especially high sympathetic tone at baseline and/or attenuated reactivity or variability to stress, appears to be involved in disturbance of emotional and interoceptive awareness. (3) Several studies suggest that a link or a cooperative relationship exists between emotional and somatic awareness, and that somatic awareness is the more fundamental of the two types of awareness. Emotional awareness, somatic awareness and autonomic homeostatic processing generally occur in parallel or concurrently. However, some complex features of pathologies include coexistence of reduced interoceptive awareness and somatosensory amplification. The autonomic homeostatic process is fundamentally involved in emotional and somatic awareness. Investigation of these types of awareness with both neuroimaging evaluations and estimation of peripheral autonomic function are required as next steps for exploration of the relationship between awareness and human somatic states including somatic symptoms as well as general psychosomatic health.
\end{abstract}

Keywords: Emotional awareness, Alexithymia, Somatic awareness, Interoception, Interoceptive awareness, Alexisomia, Autonomic function, Homeostasis, Physiological, Psychosomatic medicine

\section{Background}

Emotional awareness and somatic awareness are essential processes for human psychosomatic health, because disturbance of these types of awareness leads to unhealthy conditions through obstruction of homeostatic processing. Emotional/somatic awareness is the state in which individuals have access to their own emotional/ somatic condition [1]. A typical trait of lacking emotional awareness related to psychosomatic symptoms is

\footnotetext{
* Correspondence: kanbara-psim@umin.ac.jp

Department of Psychosomatic Medicine, Kansai Medical University, 2-5-1, Shinmachi, Hirakata, Osaka 573-1010, Japan
}

called alexithymia. In contrast, somatic awareness is physiologically based on interoception, which is defined as the homeostatic afferent neural system that represents the physiological condition of the body in humans [2-4]. Alexisomia is a term that, in contrast with alexithymia, refers to a trait of lacking somatic awareness [5]. The mechanisms that link these traits to unhealthy conditions include implicit emotional processing [6-8], disconnection between neocortical and subcortical systems $[9,10]$, and homeostatic inadequacy by blunt interoception $[11,12]$ as will be described later. In the present paper, we focus on the relationships among emotional 
awareness, somatic awareness, and autonomic homeostatic processing.

Patients with psychosomatic disorders often present with difficulties in awareness and expression of their emotions, or alexithymia trait [13]. The alexithymia construct was conceptualized by Nemiah, Freyberger, and Sifneos $[14,15]$ as a trait characterized by difficulty in identifying feelings, difficulty in describing feelings, externally oriented thinking, and a limited capacity of imagination. The alexithymia trait is involved in generation and expansion of somatic symptoms and related not only to psychosomatic disorders but also to several physical illnesses [16, 17], functional somatic disorders such as functional gastrointestinal disorders [18] or so-called "medically unexplained symptoms" [19], chronic pain $[20,21]$ and certain types of illness behavior [22]. Better understanding of alexithymic features for patients with psychosomatic or functional somatic disorders, in both general medical settings and specialized clinical settings, is required.

Links between the emotional process and the homeostatic physiological process are important for understanding the relationship of alexithymia to somatic symptoms or disorders. Lane conceptualized the explicit and implicit emotional processes and demonstrated that alexithymia is one of the constructs related to implicit emotional process [6-8]. Explicit emotional processing in this context means that negative emotional states such as depression, anxiety and hostility are associated with the unhealthy state or disease, while implicit emotional processing means interruption in the awareness and expression of negative emotion, which leads to unhealthy physiological conditions, typically psychosomatic disorders.

They also advocated the level of emotional awareness model [6], in which greater awareness corresponds to differentiated emotion and less awareness corresponds to undifferentiated emotion or somatic sensation. According to the concept and the model, verbal expressions are accessible for well-differentiated emotions, while undifferentiated emotions tend to lead to physiological dysfunctions or somatic symptoms through implicit emotional processing.

MacLean hypothesized for the first time that interference in connections between the limbic system and the neocortical system is the basic source of psychosomatic problems [23, 24]. Emotional awareness, in this context, is an emotional processing into awareness enabled by connection between the neocortical and subcortical systems. Alexithymia is therefore assumed to be one of the disconnection syndromes $[7,9,10]$. Disconnection between the higher-level neocortical emotional processes and subcortical emotion-generating processes contributes to dysfunctions in the autonomic nervous system
(ANS) and hypothalamic-pituitary-adrenal (HPA) axis [23], and hence leads to homeostatic dysregulation and finally to disease.

\section{Neural substrates for emotional/somatic awareness and autonomic homeostatic processing}

Neuroimaging approaches have demonstrated several neural substrates for the implicit emotional and somatic processes and their links to homeostatic regulation.

The amygdala is a core limbic structure, and many studies have demonstrated its functions in emotional processing [25-28] and emotion-related memory [29, 30]. The cingulate cortex is a paralimbic structure, and the anterior cingulate cortex (ACC) was initially thought to have general affective and emotional functions [31, 32]. It is now recognized to have two subdivisions: the dorsal and ventral portions of the ACC. Although the dorsal ACC (dACC) has generally been thought to have primarily cognitive functions [33, 34], the dACC actually plays considerable roles in emotional processing $[35,36]$. Moreover, several bodies of evidence using emotional awareness scales [37] for example suggest the dACC is involved in emotional awareness or expression of emotion [38, 39]. Whereas, the ventral ACC mainly connects to the amygdala, hypothalamus and insula [33, 40], it has outflow to autonomic nervous and endocrine systems, and plays a role in regulation of emotional responses with respect to limbic systems [39, 41, 42]. Overall, the amygdala is associated primarily with unconscious or implicit emotional processing, while the cingulate cortex is associated predominantly with conscious processing of emotion [43, 44].

On the other hand, the insula is a cortical area closely connected with the limbic system, which receives somatosensory signals [45]. The insula is particularly involved in interoception or interoceptive awareness $[2,3$, 46]. Interoceptive stimuli include thirst, dyspnea, sensual touch, coolness, warmth, heartbeat, and so on [4]. Interoceptive representations in neural substrates are primarily via the anterior insula $[4,46]$, which has a fundamental role in all subjective feelings from the body [4], while the ACC is responsible for autonomic alteration along with interoception [47]. The basic purpose of interoceptive awareness is to optimize homeostatic efficiency [11]. Disturbed interoceptive awareness therefore could lead to an unhealthy condition through homeostatic insufficiency. The relationship between interoceptive and emotional awareness has been studied and several lines of evidence to be described later have suggested a cooperative relationship between the two.

Most of these neural substrates for implicit emotional processing and interoceptive awareness are also known to be responsible for the ANS and HPA axis functions, which are major homeostatic regulatory systems or 
allostatic systems [48, 49]. Allostasis is the process of achieving stability through physiological or behavioral change; the set point of the stability is not static but dynamic in allostatic systems [48]. Alteration of the set point could lead to disease; this constitutes one explanation for the psychosomatic process. Hence, the concept is important for psychosomatic health.

Damasio advocated a neural model, in which the neural distinctions among primary emotion, secondary emotion, and feeling are demonstrated [50]. Primary emotions are the result of an implicit subconscious emotional process that occurs as a first response to a situation, and are closely related to adaptive and survival processes; in contrast, secondary emotions are the outcome of a process that occurs after the primary emotions, and represent social and higher-level processes with the experience of consciously processed emotion. The primary emotional response is considered the phylogenetically older behavioral and a physiological expression of the emotional response [7]. Meanwhile, interoception is a homeostatic afferent pathway and sensory aspects of homeostasis that represent the physiological condition of the body [2]. Thus, the implicit emotional and interoceptive processes have a basal relationship with the homeostatic process through ANS and HPA axis.

Considering these theories and arguments together, both emotional awareness and somatic interoceptive awareness are essential processes for human psychosomatic health. The homeostatic physiological process-that is, autonomic and HPA functions, or its response to environment or stress-is intimately linked to emotional and interoceptive awareness; therefore, this process is one of the most important clues to investigate both types of awareness. The ANS is key for achieving the appropriate modification of physiological parameters, although the hormonal system is activated simultaneously [51]. Additionally, the autonomic indexes are easy to measure and easy to apply in psychophysiological approaches such as biofeedback to psychosomatic patients. We have therefore directed attention to autonomic response to stress as a homeostatic marker. Here, the purpose of the present paper is to review evidence of the links among emotional awareness, somatic interoceptive awareness and autonomic homeostatic processing.

\section{Emotional awareness and autonomic homeostatic processing}

Many studies have addressed autonomic characteristics in response to stress tasks or various stimuli in alexithymic individuals. The findings vary by experimental condition and have some inconsistencies [52]. Some studies indicate a hyperarousal model of alexithymia, in which higher autonomic responses are observed in alexithymic individuals $[53,54]$, whereas considerable studies support a hypoarousal model of alexithymia, in which attenuated autonomic reactivity inhibits the perception of emotional signals [55-57]. These inconsistencies are probably due in part to differences in stimuli (e.g., tasks involving mental arithmetic, affective picture viewing, or social speech stress) and differences in physiological measurements (e.g., heart rate or other cardiovascular marker, electrodermal activity or skin conductance level, or muscle tension).

Although results have diverged in some areas, two findings are mostly consistent. (1) Autonomic tone at baseline, as measured by electrodermal activities such as skin conductance, is higher in alexithymic than in nonalexithymic individuals, whereas responses to stress (measured as change scores from baseline) are relatively low [53, 54, 58-60]. (2) In contrast, baseline autonomic states, measured by heart rates, are unrelated to alexithymic levels, while the responses to stress differ according to stimulus types $[58,59,61,62]$.

Electrodermal activities are known to be an index of autonomic tone related to emotional arousal that is mainly controlled by the sympathetic system [63], while cardiac activities are regulated by both sympathetic and vagal functions. Electrodermal activities are also known to follow the "law of initial value," which means that high baseline levels limit the amount of change that can be produced by stimuli [64]. Another study using cortisol levels suggested increased basal HPA activity in alexithymic subjects [65]. The HPA axis and sympatheticadrenal-medullary systems are major stress-responsive systems that work in alliance and react concurrently in most cases with stressful situations [66]. On the basis of these reviews, it is plausible that heightened resting sympathetic tone and/or attenuated reactivity to stress, or variability, are involved in the disturbance of proper perception of emotional inputs in a majority of alexithymic individuals (Fig. 1).

Additionally, several studies [65, 67] have reported varying autonomic or HPA reactivity results with different subscales of the 20-item Toronto Alexithymia Scale (TAS-20) [68, 69]. For example, Pollatos et al. demonstrated that only the alexithymia facet "difficulty in describing feelings" was associated with smaller electrodermal responses [67]. Bermond and Vorst presented evidence that the alexithymia trait has both affective and cognitive dimensions that are orthogonal to one another, and proposed different physiological features in these dimensional categories [70, 71]. Therefore, such heterogeneity of alexithymia construct should also be considered as a source of the variety in outcomes of the studies described earlier.

Our previous studies in patients with psychosomatic or functional somatic symptoms, who generally have 


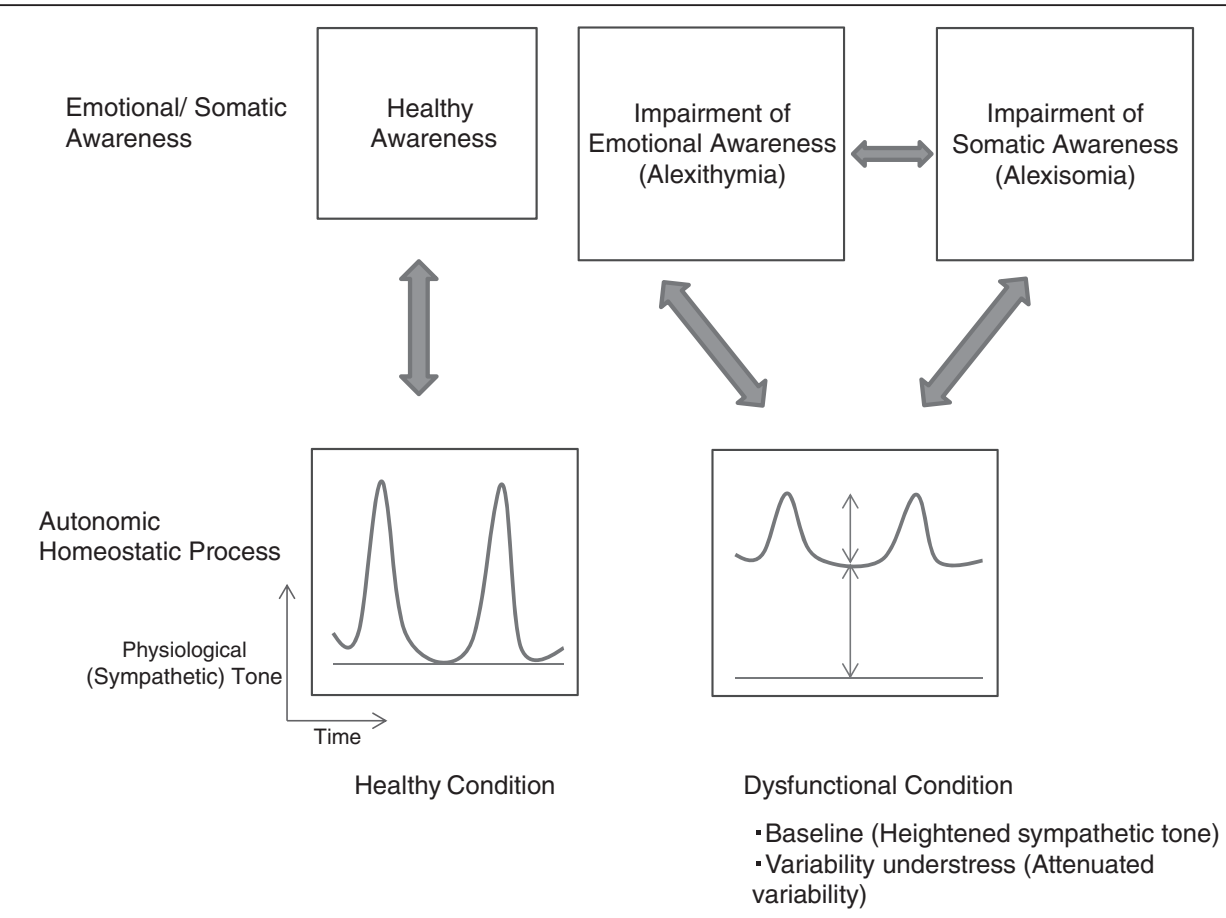

Fig. 1 Hypothesis of links among emotional awareness, somatic awareness and autonomic homeostatic processing. Autonomic dysfunction, especially heightened sympathetic tone at baseline and/or impaired variability or reactivity to stress, is involved in the disturbance of emotional and interoceptive awareness. In a dysfunctional condition of the autonomic nervous system, low variability of physiological tone contributes to disturbance of proper perception in emotional/somatic inputs, and the disturbance leads to the impairment of emotional and/or somatic awareness

been thought to have difficulties in emotional and/or somatic awareness, showed overall hyporeactivity to a mental arithmetic stress task in several physiological measurements including skin conductance level, skin temperature, and blood volume pulse amplitude [72, 73]. A subsequent study with a larger sample identified two clusters of psychophysiological stress response patterns: a majority cluster whose members have low stress response patterns and another cluster with high or even response patterns [74]. Another study suggested that functional somatic syndrome patients with difficulty in identifying feelings had higher sympathetic tone at pre-stress baseline, measured by salivary amylase [75, 76]. Moreover, the alexithymic trait and resting sympathetic tone correlated positively in healthy controls [75].

Considering these findings and aforementioned reviews together, several dysfunctional states in ANS are involved in the disturbance of emotional awareness; especially, high sympathetic tone at baseline and/or impaired autonomic variability affect emotional awareness in a number of alexithymic individuals (Fig. 1). Whereas, the alexithymia construct is heterogeneous, and one or more other mechanisms could also affect emotional awareness in other types of alexithymia.

\section{Emotional awareness and feedback model through vagal function}

Several studies have indicated that the function of the medial prefrontal cortex, which has a strong connection to limbic structures such as the amygdala, is correlated positively with autonomic vagal activity measured by the vagal component of heart rate variability [77-80]. The ACC, which is anatomically adjacent to the medial prefrontal cortex, has also been shown to have a positive correlation with vagal function [81].

The medial prefrontal cortex and the ACC have a role of conscious processing of emotion as described previously $[7,8]$. Conscious processing or conscious awareness of emotion is generally accepted clinically as an important process for emotional self-regulation [82]. Lane et al. argued that conscious processing of emotion requires the transmission of subcortical affective information to the cerebral cortex - medial prefrontal cortex and ACC in this context [80]. The processing also requires top-down feedback from cortical to subcortical function [43, 80]. They considered the positive correlation of the cortical activity with vagal function described previously as evidences of top-down feedback $[7,80]$.

Based on the existing evidence and its interpretations, the conscious processing of emotion is assumed to have 
a negative feedback loop through vagal function $[6,83]$; that is, when emotional experience is consciously processed into awareness, vagal tone is accelerated, after which emotional arousal is regulated [7]. In other words, the medial prefrontal cortex and ACC have a tonic inhibitory effect through vagal activity when these areas are activated in connection with conscious emotional experience. Vagal tone has been considered to have a function of regulating emotional responses [84], or selecting an optimal response and inhibiting less optimal ones [80] (Fig. 2).

Research suggests that the neural pathway for the feedback process involves cortical areas, from the ventrolateral prefrontal cortex and especially the medial prefrontal cortex; through limbic system structures such as the cingulate cortex (especially the ACC), amygdala and hypothalamus; to the ANS [7, 47, 83, 85] (Fig. 2). The insula is also involved in this pathway in addition to being a representative center for interoception as described before. The anterior insula and ACC have a close functional relationship and the two areas conjointly bear an important role in emotional processing [86].

If conscious processing is disturbed, vagal tone would not be accelerated, and the resting sympathetic tone would be easily activated. This negative feedback model suggests a mechanism of the relationship between the autonomic function and emotional awareness, and supports the hypothesis shown in Fig. 1.

\section{Interoceptive awareness and autonomic homeostatic processing}

Autonomic activity or reactivity to stress is involved in interoceptive awareness as well as emotional awareness. Some studies have suggested that individual difference in sympathetic cardiac activity affect interoceptive sensitivity to heartbeats $[87,88]$. Several subsequent studies with heartbeat perception tasks $[89,90]$ have indicated that cardiac interoceptive awareness is affected by autonomic reactivity to some tasks [91-93]. These studies suggested that interoceptive awareness is positively related to the autonomic response to such tasks.

Herbert in particular established that cardiac awareness was associated with greater sympathetic reactivity during mental stress and greater vagal reactivity during emotional picture viewing [93]. Both sympathetic and vagal activities could contribute to cardiac awareness [93]. Autonomic function or reactivity therefore affects not only emotional awareness but also somatic interoceptive awareness.

\section{Interoceptive awareness and emotional awareness}

James first described the idea that emotional experience necessarily included perception of a somatic response [94, 95], and Lange proposed a similar concept [96]. Since then, historical debates on the relationship between emotional experience and somatic change have

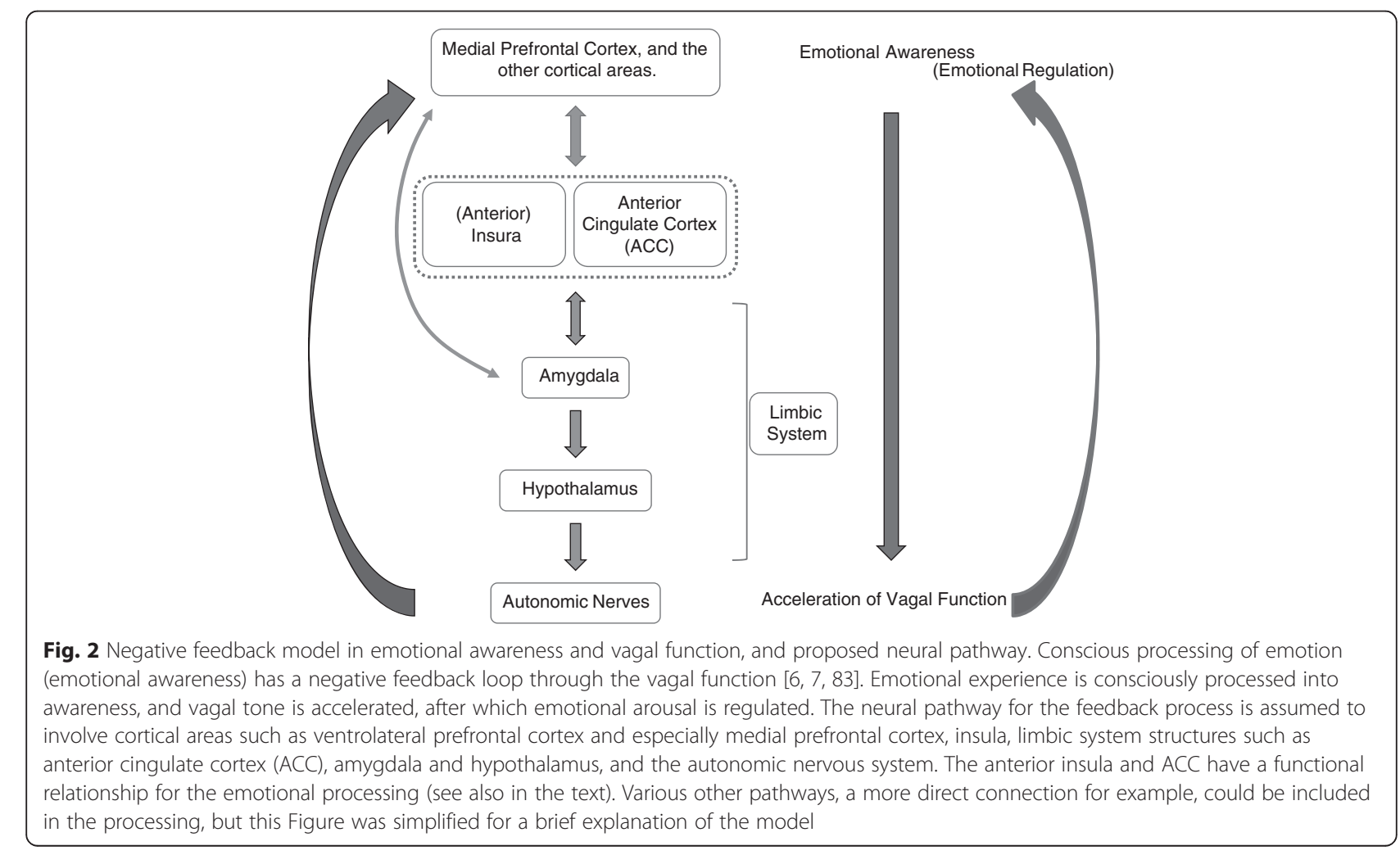


continued and have influenced theories of interaction between emotions and the body.

Considerable studies with a heartbeat perception task as an interoceptive index have demonstrated that subjective emotional experience relates positively to interoceptive sensitivity $[46,97,98]$. Consistent with these findings, evidence exists that alexithymic individuals have less interoceptive awareness [99]. Several studies using electroencephalogram (EEG) recordings also suggest interoception correlates positively with emotion processing, measured as P300 event-related potentials, for example [100-102].

Studies using heartbeat-evoked brain potential, which is observed by EEG promptly after the R-wave of the electrocardiogram over the somatosensory cortex and the frontal/prefrontal cortex, have also examined cortical processing of signals from cardiovascular activities [103, 104]. The heartbeat-evoked potential could be a plausible neurophysiological marker of cardiac interoceptive awareness [105] and has a potential significance for exploration of the relationship between cardiac interoception and cortical awareness processes. Heartbeat-evoked potentials have been linked to empathy [106], suggesting a correlation between cardiac awareness and emotional processing.

The anterior insula, a core substrate for interoception, is also activated by emotional processing [107, 108]. Neuroimaging studies suggested that the common activated areas for interoceptive awareness and emotional experience were the right anterior insular cortex and ventromedial prefrontal cortex $[109,110]$. These findings suggest the close neurophysiological correlation of emotional and somatic interoceptive awareness. Additionally, difficulties in emotional awareness, i.e., alexithymia, are in most cases clinically associated with difficulties in somatic awareness, or alexisomia [5].

In contrast, several studies have suggested activation of some somatosensory systems in alexithymic individuals. For example, visceral sensitivity to stimulation is associated with alexithymia [111]. Alexithymic persons have exhibited higher activation in the pain matrix area of brain [112]. Women with alexithymia have demonstrated higher activation of sensory and motor cortex, compared with controls [113]. These findings are consistent with the psychosomatic theory in which alexithymic patients tend to express their conflicts of emotion somatically rather than linguistically.

It is seemingly contradictory that emotional and somatic interoceptive awareness positively correlate, while impairment of emotional awareness leads to somatosensory amplification. Moriguchi and Komaki elucidated this argument by demonstrating the limitation of TAS20 as a self-report measure and the heterogeneity of alexithymia [114]. As mentioned before, Bermond and
Vorst hypothesized subtypes of alexithymia by dimensional model: one characterized by attenuation of physiological arousal, and another marked by intact physiological arousal [70]. This typology could partially explain the apparent contradiction.

Another explanation is suggested by the difference between interoceptive awareness and somatosensory amplification [115]. For example, a number of patients with chronic pain, who have less emotional and interoceptive awareness, experience persistence of a specific type of pain, such as myalgia of some part of the body, with somatosensory amplification. Barsky et al., who advocated the concept of somatosensory amplification, demonstrated that patients with hypochondria had high somatosensory amplification but were not good at cardiac awareness [116, 117]. Moreover, Mailloux and Brener directly examined the relationship between somatosensory amplification and cardiac interoceptive awareness [118]. They showed greater somatosensory amplification in poor heartbeat detectors than in good detectors, and suggested that somatosensory amplification is a "cognitive bias" and does not reflect heightened somatic awareness.

Interoceptive awareness and somatosensory amplification are therefore not on the same axis. At its core, somatosensory amplification is a condition in which sensation of a somatic state is higher than the assumed actual state, whereas interoception is an "accurate" sensation of a somatic state; therefore, reduced interoception could mean either a hyper- or hyposensitive condition. Individuals with less interoceptive or emotional awareness may not perceive their own somatic or emotional state properly and may exhibit somatosensory amplification.

According to psychosomatic theory, the amplification comes not only from cognitive bias but also from physiological bias. Kano and Fukudo reported in their recent review that alexithymic individuals showed reduced responses in limbic areas in their cognitive processing, but amplified responses in more primitive processing, e.g., hypersensitivity to visceral pain [119]. Somatosensory amplification in more primitive processes may occur typically in psychosomatic patients who have less emotional and interoceptive awareness.

\section{Discussion}

Several key points were presented in this paper $^{1}$. First, both emotional and somatic interoceptive awareness are important for psychosomatic health. The main subcortical neural substrates for these processes are limbicrelated systems: the amygdala, cingulate and insula, which are also responsible for autonomic and HPA axis functions for optimization of homeostatic efficiency. Second, considerable studies show that autonomic 
activity and/or reactivity to stress correlates with both emotional and interoceptive awareness. The hypothesis that autonomic dysfunction is involved in the disturbance of emotional and interoceptive awareness has been advocated (Fig. 1). Third, several sources of evidence suggest links or a cooperative relationship between emotional and somatic awareness. However, impairment of emotional or somatic awareness often accompanies somatosensory amplifications in a specific area or system, typically in psychosomatic patients.

The first point suggests links between emotional/interoceptive awareness and psychosomatic health through homeostatic or allostatic process. The so-called emotional awareness or somatic awareness approach, which is assumed to accelerate emotional or somatic awareness, could also accelerate the homeostatic process and lead to psychosomatic health. One of the key mechanisms for psychosomatic health is links between limbicrelated systems and higher cortical systems. The emotional/somatic awareness approaches are assumed to accelerate these links.

\section{Awareness and autonomic function: Clinical implications}

Regarding the second point, in which both emotional and somatic awareness correlate with autonomic function, increased awareness could improve autonomic function and consequently autonomic-related symptoms; or reciprocally, the improvement of autonomic function could facilitate awareness. A number of neuroimaging studies have demonstrated that putting emotional feeling into words, i.e., affect labeling, changed the response to stimuli of the amygdala and other limbic structures [85, 120]. These results suggest that conscious awareness and linguistic processing of emotion effect alterations in autonomic function. Although it is inconclusive whether emotional awareness or linguistic processing actually effects the alteration of limbic function, the linguistic process necessarily involves conscious awareness, and both processes could improve autonomic function. The aforementioned negative feedback model, in which conscious awareness of emotion accelerates vagal function [6, 83] (Fig. 2) supports these arguments.

Interoceptive awareness also could improve autonomic function in a way suggested by the second and third points and arguments in the last two sections. In the context of the negative feedback model, interoceptive awareness probably accelerates vagal function, which leads to the reduction of symptoms. Actually, Schaefer et al. reported that improving interoceptive awareness reduced symptom distress in patients with somatoform disorders or medically unexplained symptoms [121, 122]. Further empirical verification of the relationship between interoceptive awareness and the vagal feedback process is required.
On the basis of this review, the hypothesis presented in Fig. 1, and our previous studies, in which psychosomatic patients had low autonomic responsiveness, we conclude that autonomic responsiveness is involved in one of the most important processes for emotional/somatic awareness upon psychosomatic health. Our previous study also suggested co-occurrence of low variability in subjective feelings of tension and attenuated autonomic response [72]. Low variability in autonomic function and subjective feeling probably contributes to disturbance of emotional and/or interoceptive awareness through less discriminative sensation. Excessive variability also may contribute to this disturbance. We therefore tentatively consider variability in autonomic function as one of the most important contributing factors for emotional/somatic awareness although this hypothesis needs further verification study.

The autonomic dysfunctional condition interacting with the impairment of emotional and/or somatic awareness seen in Fig. 1 leads to unhealthy psychosomatic conditions through insufficient homeostatic or allostatic process along with autonomic dysfunction. The condition should be ameliorated by improvement in emotional/somatic awareness.

\section{Alexithymia and alexisomia}

Regarding the third point, either the emotional or somatic form of awareness could facilitate the other. The concept of "response system coherence" [123-125], that is, the idea that emotions organize and synchronize different response systems (e.g., behavioral and physiological) [126], can explain the cooperative relationship between emotional and somatic awareness. Sze et al. reported that the coherence between subjective emotion and cardiac awareness was greater in those who had specialized training in promotion of somatic awareness than did controls [126]. Our arguments are consistent with the clinically accepted process that "body-oriented" approaches such as yoga, biofeedback, and certain kinds of body psychotherapies encourage the emotional process of awareness, i.e., improvement of the alexithymic trait.

Ikemi, a founder of psychosomatic medicine in Japan, first described the concept of alexisomia in association with alexithymia in the early 1980s as clinical characteristics of difficulties in awareness or expression of somatic feeling/sensations from an Eastern point of view [5, 127]. Mind and body are considered as one harmonic entity in the Eastern view, but as dualistic entities in the Western view. The concept of alexisomia was developed on the basis of the Eastern conceptualization, but the concept has not yet been explored extensively. Meanwhile, the mechanism of interoceptive awareness has been investigated in the context of neurophysiological studies as described in the present paper. Impairment of 
interoceptive awareness appears to be similar to alexisomia, although a conclusive decision about whether these states are exactly the same cannot yet be made.

Regarding the relationship between alexisomia and alexithymia, Moriguchi and Komaki addressed this topic with reference to reviews of neuroimaging studies and concluded that somatic awareness is the basis of emotional awareness because bodily states, including autonomic and hormonal status, are fundamentally involved in basic affective states [114]. Damasio advocated the somatic marker hypothesis, wherein emotional processes lead behavior, especially the choice of a proper action or decision, and demonstrated the fundamental role of the physiological state in emotional processing $[50,128]$.

Therefore, although emotional awareness, somatic awareness and autonomic processing are generally parallel or concurrent processes, the aforementioned arguments suggest that somatic awareness is fundamental for emotional awareness, and that the autonomic homeostatic process has a more fundamental involvement in somatic and emotional awareness. However, psychosomatic patients with alexithymia/alexisomia have complex pathologies, which include coexistence of impairment of interoceptive awareness and somatosensory amplification, as previously described. This complexity might be associated with the multiple pathologies seen in patients not only with psychosomatic disorder but also with chronic pain or functional somatic syndromes.

Another source of complexity is that the process of awareness is involved in various levels of processing such as peripheral/sensory, intermediate/limbic related and central/cognitive systems. Alexisomia includes impairments of not only peripheral processes but also cognitive or even higher-level processes [5]. One of the challenges and difficulties for investigation of mechanisms of alexisomia is that this multilevel construct applies here as well as in alexithymia. Further clinical and physiological investigations that consider such multilevel properties are needed for better understanding of the process of alexisomia and interoceptive awareness.

\section{Self and awareness}

Finally, any arguments about awareness, which can be seen as a change in the subjective-objective relationship, must include some mention of the notion of "self", because subjective feelings necessarily require a self that experiences the feelings. Craig stated that to have awareness is to know that one exists [4] and addressed the neural model for integrative representations of all feelings from the body at any moment as "the sentient self" [11]. Damasio posited that the self is a repeatedly reconstructed biological state, called "neural self" [129]. Thus, the self is inseparable from the somatic and emotional feelings or senses and awareness is a state of linkage between the self and the senses. This theme is extremely profound and beyond the scope of our paper, but clinically, impairment of emotional/somatic awareness likely relates to disestablishment of the self. We may therefore have to consider the need for reestablishment of the self in our approach to patients with difficulties in these types of awareness or alexithymia/alexisomia, especially children or persons with developmental disorders.

\section{Conclusions}

This paper reviewed links between emotional awareness, somatic awareness including interoception, and autonomic homeostatic processing, and advocated a hypothesis about the links between the two types of awareness and autonomic function. Autonomic homeostatic processing has a fundamental involvement in both emotional and somatic awareness. Investigation of these types of awareness, using both neuroimaging evaluations and estimation of peripheral autonomic function, are required next steps for exploration of their relationship to somatic symptoms, and to the more general issue of human psychosomatic health.

\section{Endnote}

${ }^{1}$ For readability, the key points of this manuscript are indicated as (1), (2), and (3) in the abstract. The numbers correspond to points labeled as "first," "second," and "third" in the Discussion section. The first point also relates to the context in the Background and to the subsection titled "Neural substrates for emotional/ somatic awareness and autonomic homeostatic processing." The second point corresponds to the following subsections in the main text: "Emotional awareness and autonomic homeostatic processing," "Emotional awareness and feedback model through vagal function," and "Interoceptive awareness and autonomic homeostatic processing." The third point corresponds to the subsection titled "Interoceptive awareness and emotional awareness."

\section{Abbreviations}

FSS: functional somatic syndrome; ANS: autonomic nervous system; HPA (axis): hypothalamic pituitary adrenal (axis); (d) ACC: (dorsal) anterior cingulate cortex; TAS-20: 20-item Toronto Alexithymia Scale; EEG: electroencephalogram.

Competing interests

The authors declare that they have no competing interests.

\section{Authors' contributions}

KK and MF collected the research and edited the paper. Both authors read and approved the final manuscript.

\section{Acknowledgements}

This review study was supported in part by Grant from Pfizer Health Research Foundation and the Japan Society for the Promotion of Science, Grant-in-Aid for Scientific Research (C), 22570228, 2010-2012.

We would like to express our appreciation to Prof. Haruhiko Murakawa, Prof. Kiyoshi Hamano and Dr. Makoto Hashizume for their valuable suggestions. We would also like to sincerely thank the members of staff at Kansai Medical University, and members of the Society for Alexisomia Research. 


\section{Received: 11 August 2015 Accepted: 11 February 2016 Published online: 10 May 2016}

\section{References}

1. Chalmers DJ. The Conscious Mind. In: Search of a Fundamental Theory. 1st ed. New York: Oxford University Press; 1997.

2. Craig AD. How do you feel? Interoception: the sense of the physiological condition of the body. Nat Rev Neurosci. 2002;3:655-66.

3. Craig AD. Interoception: the sense of the physiological condition of the body. Curr Opin Neurobiol. 2003;13:500-5.

4. Craig AD. How do you feel-now? The anterior insula and human awareness. Nat Rev Neurosci. 2009;10:59-70.

5. Ikemi $Y$, Ikemi A. An oriental point of view in psychosomatic medicine. Psychother Psychosom. 1986;45:118-26.

6. Lane RD, Schwartz GE. Levels of emotional awareness: a cognitivedevelopmental theory and its application to psychopathology. Am J Psychiatry. 1987;144:133-43.

7. Lane RD. Neural substrates of implicit and explicit emotional processes: a unifying framework for psychosomatic medicine. Psychosom Med. 2008;70:214-31.

8. Lane RD. Theory of emotional awareness and brain processing of emotion Int Congr Ser. 2006;1287:116-21.

9. Geschwind N. Disconnexion syndromes in animals and man. I. Brain. 1965;88:237-94.

10. Geschwind N. Disconnexion syndromes in animals and man. II. Brain. 1965:88:585-644.

11. Craig AD. The sentient self. Brain Struct Funct. 2010;214:563-77.

12. Duschek S, Montoro Cl, Reyes Del Paso GA. Diminished interoceptive awareness in fibromyalgia syndrome. Behav Med. 2015;4289:Oct 2:0 [Epub ahead of print].

13. Sifneos PE. The prevalence of "alexithymic" characteristics in psychosomatic patients. Psychother Psychosom. 1973;22:255-62.

14. Nemiah J, Freyberger $\mathrm{H}$. Alexithymia: a view of the psychosomatic process In: Hill O, editor. Modem trends in psychosomatic medicine, vol. 3. London: Butterworths; 1976. p. 430-9.

15. Nemiah J, Sifneos P. Affect and fantasy in patients with psychosomatic disorders. In: Hill O, editor. Modem trends in psychosomatic medicine, vol. 2. London: Butterworths; 1970. p. 26-34

16. Taylor GJ, Bagby RM, Parker JDA. Disorders of affect regulation: Alexithymia in medical and psychiatric illness. Cambridge: Cambridge University Press; 1997.

17. Grabe HJ, Schwahn C, Barnow S, Spitzer C, John U, Freyberger HJ, et al. Alexithymia, hypertension, and subclinical atherosclerosis in the general population. J Psychosom Res. 2010;68:139-47.

18. Porcelli P, De Carne M, Todarello O. Prediction of treatment outcome of patients with functional gastrointestinal disorders by the diagnostic criteria for psychosomatic research. Psychother Psychosom. 2004;73:166-73.

19. Kooiman CG. The status of alexithymia as a risk factor in medically unexplained physical symptoms. Compr Psychiatry. 1998;39:152-9.

20. Acklin MW, Bernat E. Depression, alexithymia, and pain prone disorder: a Rorschach study. J Pers Assess. 1987:51:462-79.

21. Lumley MA, Radcliffe AM, Macklem DJ, Mosley-Williams A, Leisen JCC, Huffman $J$, et al. Alexithymia and pain in three chronic pain samples: comparing Caucasians and African Americans. Pain Med. 2005;6:251-61.

22. Lumley MA, Stettner L, Wehmer F. How are alexithymia and physical illness linked? A review and critique of pathways. J Psychosom Res. 1996:41:505-18.

23. MacLean PD. Psychosomatic disease and the visceral brain; recent developments bearing on the Papez theory of emotion. Psychosom Med. 1949;11:338-53

24. MacLean PD. Some psychiatric implications of physiological studies on frontotemporal portion of limbic system (visceral brain). Electroencephalogr Clin Neurophysiol. 1952:4:407-18.

25. Weiskrantz L. Behavioral changes associated with ablation of the amygdaloid complex in monkeys. J Comp Physiol Psychol. 1956;49:381-91.

26. Aggleton JP. The Amygdala: A Functional Analysis. 2nd ed. New York: Oxford University Press; 2000.

27. LaBar KS, LeDoux JE, Spencer DD, Phelps EA. Impaired fear conditioning following unilateral temporal lobectomy in humans. J Neurosci. 1995;15:6846-55.

28. Bechara A, Tranel D, Damasio H, Adolphs R, Rockland C, Damasio AR C. Double dissociation of conditioning and declarative knowledge relative to the amygdala and hippocampus in humans. Science. 1995;269:1115-8.
29. Babinsky R, Calabrese P, Durwen HF, Markowitsch HJ, Brechtelsbauer D, Heuser $\mathrm{L}$, et al. The possible contribution of the amygdala to memory. Behav Neurol. 1993;6:167-70.

30. Adolphs R, Cahill L, Schul R, Babinsky R. Impaired declarative memory for emotional material following bilateral amygdala damage in humans. Learn Mem. 1997:4:291-300.

31. Papez JW. A proposed mechanism of emotion. Arch Neurol Psychiatry. 1937;38:725.

32. Vogt BA, Finch DM, Olson CR. Functional heterogeneity in cingulate cortex: the anterior executive and posterior evaluative regions. Cereb Cortex. 1992;2:435-43.

33. Bush G, Luu P, Posner M. Cognitive and emotional influences in anterior cingulate cortex. Trends Cogn Sci. 2000;4:215-22.

34. Mohanty A, Engels AS, Herrington JD, Heller W, Ringo Ho M-HH, Banich MT, et al. Differential engagement of anterior cingulate cortex subdivisions for cognitive and emotional function. Psychophysiology. 2007:44:343-51.

35. Phan KL, Wager T, Taylor SF, Liberzon I. Functional neuroanatomy of emotion: a meta-analysis of emotion activation studies in PET and fMRI. Neuroimage. 2002:16:331-48.

36. Hutcherson CA, Goldin PR, Ochsner KN, Gabrieli JD, Barrett LF, Gross JJ. Attention and emotion: does rating emotion alter neural responses to amusing and sad films? Neuroimage. 2005;27:656-68.

37. Lane RD, Quinlan DM, Schwartz GE, Walker PA, Zeitlin SB. The levels of emotional awareness scale: a cognitive-developmental measure of emotion. J Pers Assess. 1990;55:124-34.

38. McRae K, Reiman EM, Fort CL, Chen K, Lane RD. Association between trait emotional awareness and dorsal anterior cingulate activity during emotion is arousal-dependent. Neuroimage. 2008:41:648-55.

39. Etkin A, Egner T, Kalisch R. Emotional processing in anterior cingulate and medial prefrontal. Trends Cogn Sci. 2011;15:85-93.

40. Devinsky O, Morrell MJ, Vogt BA. Contributions of anterior cingulate cortex to behaviour. Brain. 1995:118(Pt 1):279-306.

41. Cabeza R, Nyberg L. Imaging cognition: an empirical review of PET studies with normal subjects. J Cogn Neurosci. 1997:9:1-26.

42. Drevets WC, Raichle ME. Suppression of regional cerebral blood during emotional versus higher cognitive implications for interactions between emotion and cognition. Cogn Emot. 1998;12:353-85.

43. Williams LM, Liddell BJ, Kemp AH, Bryant RA, Meares RA, Peduto AS, et al. Amygdala-prefrontal dissociation of subliminal and supraliminal fear. Hum Brain Mapp. 2006;27:652-61.

44. Morris JS, Ohman A, Dolan RJ. Conscious and unconscious emotional learning in the human amygdala. Nature. 1998;393:467-70.

45. Fabri M, Burton $\mathrm{H}$. Ipsilateral cortical connections of primary somatic sensory cortex in rats. J Comp Neurol. 1991;311:405-24.

46. Critchley HD, Wiens S, Rotshtein P, Ohman A, Dolan RJ. Neural systems supporting interoceptive awareness. Nat Neurosci. 2004;7:189-95.

47. Critchley HD. Neural mechanisms of autonomic, affective, and cognitive integration. J Comp Neurol. 2005;493:154-66.

48. McEwen BS, Stellar E, Bruce S. Stress and the individual. Mechanisms leading to disease. Arch Intern Med. 1993;153:2093-101.

49. Sterling P, Eyer J. Allostasis: a new paradigm to explain arousal pathology. In: Fisher J, Reason J, editors. Handbook of Life Stress Cognition, and Health. New York: Wiley; 1988. p. 629-49.

50. Damasio AR. Descartes' Error : Emotion, Reason, and the Human Brain. New York: Penguin; 1994.

51. Damasio AR. Testing the somatic marker hypothesis. In: Descartes' error: emotion, reason, and the human brain. New York: Penguin; 1994. p. 205-22.

52. Taylor GJ, Bagby RM. New trends in alexithymia research. Psychother Psychosom. 2004;73:68-77.

53. Infrasca R. Alexithymia, neurovegetative arousal and neuroticism. An experimental study. Psychother Psychosom. 1997;66:276-80.

54. Rabavilas AD. Electrodermal activity in low and high alexithymia neurotic patients. Psychother Psychosom. 1987;47:101-4.

55. Wehmer F, Brejnak C, Lumley M, Stettner L. Alexithymia and physiological reactivity to emotional-provoking visual scenes. J Nerv Ment Dis. 1995;183:351-7.

56. Linden W, Lenz JW, Stossel C. Alexithymia, defensiveness and cardiovascular reactivity to stress. J Psychosom Res. 1996:41:575-83.

57. Neumann SA, Sollers JJ, Thayer JF, Waldstein SR. Alexithymia predicts attenuated autonomic reactivity, but prolonged recovery to anger recall in young women. Int J Psychophysiol. 2004;53:183-95. 
58. Friedlander L, Lumley MA, Farchione T, Doyal G. Testing the alexithymia hypothesis: physiological and subjective responses during relaxation and stress. J Nerv Ment Dis. 1997;185:233-9.

59. Roedema TM, Simons RF. Emotion-processing deficit in alexithymia. Psychophysiology. 1999;36:379-87.

60. Stone LA, Nielson KA. Intact physiological response to arousal with impaired emotional recognition in alexithymia. Psychother Psychosom. 2001;70:92-102.

61. Newton TL, Contrada RJ. Alexithymia and repression: contrasting emotionfocused coping styles. Psychosom Med. 1994;56:457-62.

62. Luminet $\mathrm{O}$, Rimé $\mathrm{B}$, Bagby RM, Taylor G. A multimodal investigation of emotional responding in alexithymia. Cogn Emot. 2004;18:741-66.

63. Dawson ME, Schell AM, Filion DL. The electrodermal system. In: Cacioppo JT, Tassinary LG, Berntson GG, editors. Handbook of psychophysiology. Thirdth ed. New York: Cambridge University Press; 2007. p. 159-81.

64. Wilder J. Stimulus and response: The law of initial value. Bristol: J. Wright; 1967

65. De Timary P, Roy E, Luminet O, Fillée C, Mikolajczak M. Relationship between alexithymia, alexithymia factors and salivary cortisol in men exposed to a social stress test. Psychoneuroendocrinology. 2008:33:1160-4.

66. Bauer AM, Quas JA, Boyce WT. Associations between physiological reactivity and children's behavior: advantages of a multisystem approach. J Dev Behav Pediatr. 2002;23:102-13.

67. Pollatos O, Werner NS, Duschek S, Schandry R, Matthias E, Traut-Mattausch $E$, et al. Differential effects of alexithymia subscales on autonomic reactivity and anxiety during social stress. J Psychosom Res. 2011;70:525-33.

68. Bagby RM, Parker JDA, Taylor GJ. The twenty-item Toronto Alexithymia scale-I. Item selection and cross-validation of the factor structure. J Psychosom Res. 1994;38:23-32.

69. Bagby RM, Taylor GJ, Parker JD. The Twenty-item Toronto Alexithymia ScaleII. Convergent, discriminant, and concurrent validity. J Psychosom Res. 1994:38:33-40

70. Vorst HCM, Bermond B. Validity and reliability of the bermond - Vorst alexithymia questionnaire. Pers Individ Dif. 2001;30:413-34.

71. Bermond B, Vorst HCM, Moormann PP. Cognitive neuropsychology of alexithymia: implications for personality typology. Cogn Neuropsychiatry. 2006;11:332-60.

72. Kanbara K, Fukunaga M, Ishino S, Takebayashi N, Nakai Y. Characteristics of psychophysiological stress responses in patients with psychosomatic disorders. Japanese J Psychosom Med. 2005;45:685-95.

73. Kanbara K, Mitani Y, Fukunaga M, Ishino S, Takebayashi N, Nakai Y. Paradoxical results of psychophysiological stress profile in functional somatic syndrome: Correlation between subjective tension score and objective stress response. Appl Psychophysiol Biofeedback. 2004;29:255-68.

74. Kanbara K, Fukunaga M, Mutsuura H, Takeuchi H, Kitamura K, Nakai Y. An exploratory study of subgrouping of patients with functional somatic syndrome based on the psychophysiological stress response: its relationship with moods and subjective variables. Psychosom Med. 2007;69:158-65.

75. Kiba T, Kanbara K, Yamamoto K, Ban I, Oka Y, Kato F, et al. The relationship of pre-stress salivary a-amylase levels to alexithymia in patients with functional somatic syndrome. Japanese J Psychosom Med. 2013;53:670-81.

76. Kiba T, Kanbara K, Ban I, Kato F, Kawashima S, Saka Y, et al. Saliva Amylase as a measure of sympathetic change elicited by autogenic training in patients with functional somatic syndromes. Appl Psychophysiol Biofeedback. 2015:40:339-47.

77. Drevets WC. Prefrontal cortical-amygdalar metabolism in major depression. Ann N Y Acad Sci. 1999;877:614-7.

78. Buchanan SL, Valentine J, Powell DA. Autonomic responses are elicited by electrical stimulation of the medial but not lateral frontal cortex in rabbits. Behav Brain Res. 1985;18:51-62.

79. Gianaros PJ, Van Der Veen FM, Jennings JR. Regional cerebral blood flow correlates with heart period and high-frequency heart period variability during working-memory tasks: Implications for the cortical and subcortical regulation of cardiac autonomic activity. Psychophysiology. 2004;41:521-30.

80. Lane RD, McRae K, Reiman EM, Chen K, Ahern GL, Thayer JF. Neura correlates of heart rate variability during emotion. Neuroimage. 2009;44:213-22.

81. Matthews SC, Paulus MP, Simmons AN, Nelesen RA, Dimsdale JE. Functional subdivisions within anterior cingulate cortex and their relationship to autonomic nervous system function. Neuroimage. 2004;22:1151-6.

82. Barrett LF, Niedenthal PM, Winkielman P. Emotion and Consciousness. New York: Guilford Press; 2005
83. Thayer JF, Lane RD. A model of neurovisceral integration in emotion regulation and dysregulation. J Affect Disord. 2000;61:201-16.

84. Appelhans BM, Luecken LJ. Heart rate variability as an index of regulated emotional responding. Rev Gen Psychol. 2006;10:229-40.

85. Lieberman MD, Eisenberger NI, Crockett MJ, Tom SM, Pfeifer JH, Way BM. Putting feelings into words: affect labeling disrupts amygdala activity in response to affective stimuli. Psychol Sci. 2007;18:421-8.

86. Medford N, Critchley HD. Conjoint activity of anterior insular and anterior cingulate cortex: awareness and response. Brain Struct Funct. 2010;214:535-49.

87. Katkin ES. Blood, sweat, and tears: individual differences in autonomic self-perception. Psychophysiology. 1985;22:125-37.

88. Eichler S, Katkin ES. The relationship between cardiovascular reactivity and heartbeat detection. Psychophysiology. 1994;31:229-34.

89. Schandry R. Heart beat perception and Emotional Experience. Psychophysiology. 1981:18:483-8.

90. Whitehead WE, Drescher VM, Heiman P, Blackwell B. Relation of heart rate control to heartbeat perception. Biofeedback Self Regul. 1977;2:371-92.

91. Pollatos $\mathrm{O}$, Herbert BM, Matthias E, Schandry R. Heart rate response after emotional picture presentation is modulated by interoceptive awareness. Int J Psychophysiol. 2007;63:117-24.

92. Pollatos O, Herbert BM, Kaufmann C, Auer DP, Schandry R. Interoceptive awareness, anxiety and cardiovascular reactivity to isometric exercise. Int J Psychophysiol. 2007:65:167-73.

93. Herbert BM, Pollatos O, Flor H, Enck P, Schandry R. Cardiac awareness and autonomic cardiac reactivity during emotional picture viewing and mental stress. Psychophysiology. 2010;47:342-54.

94. James W. The Principles of Psychology. New York: Dover Publications; 1950

95. James W. What is an emotion? Mind. 1884;9:188-205.

96. Lange CG. The mechanism of the emotions. In: Rand B, editor. The classical psychologists: selections illustrating psychology from Anaxagoras to Wundt. Boston: Houghton Mifflin; 1912. p. 672-84.

97. Ferguson ML, Katkin ES. Visceral perception, anhedonia, and emotion. Biol Psychol. 1996:42:131-45.

98. Wiens S, Mezzacappa ES, Katkin ES. Heartbeat detection and the experience of emotions. Cognition \& Emotion. 2000;14:417-27.

99. Herbert BM, Herbert C, Pollatos O. On the relationship between interoceptive awareness and alexithymia: Is interoceptive awareness related to emotional awareness? J Pers. 2011:79:1149-75.

100. Herbert BM, Pollatos O, Schandry R. Interoceptive sensitivity and emotion processing: an EEG study. Int J Psychophysiol. 2007:65:214-27.

101. Pollatos O, Kirsch W, Schandry R. On the relationship between interoceptive awareness, emotional experience, and brain processes. Brain Res Cogn Brain Res. 2005;25:948-62.

102. Pollatos O, Gramann K, Schandry R. Neural systems connecting interoceptive awareness and feelings. Hum Brain Mapp. 2007;28:9-18.

103. Schandry R, Sparrer B, Weitkunat R. From the heart to the brain: a study of heartbeat contingent scalp potentials. Int J Neurosci. 1986;30:261-75.

104. Montoya P, Schandry R, Müller A. Heartbeat evoked potentials (HEP): topography and influence of cardiac awareness and focus of attention. Electroencephalogr Clin Neurophysiol. 1993;88:163-72.

105. Schandry R, Weitkunat R. Enhancement of heartbeat-related brain potentials through cardiac awareness training. Int J Neurosci. 1990;53:243-53.

106. Fukushima H, Terasawa Y, Umeda S. Association between interoception and empathy: evidence from heartbeat-evoked brain potential. Int J Psychophysiol. 2011;79:259-65.

107. Phillips ML, Young AW, Senior C, Brammer M, Andrew C, Calder AJ, et al. A specific neural substrate for perceiving facial expressions of disgust. Nature. 1997;389:495-8.

108. Büchel C, Morris J, Dolan RJ, Friston KJ. Brain systems mediating aversive conditioning: an event-related fMRI study. Neuron. 1998;20:947-57.

109. Terasawa $Y$, Fukushima H, Umeda S. How does interoceptive awareness interact with the subjective experience of emotion? An fMRI study. Hum Brain Mapp. 2013;34:598-612.

110. Harrison NA, Gray MA, Gianaros PJ, Critchley HD. The embodiment of emotional feelings in the brain. J Neurosci. 2010;30:12878-84.

111. Kano M, Hamaguchi T, Itoh M, Yanai K, Fukudo S. Correlation between alexithymia and hypersensitivity to visceral stimulation in human. Pain. 2007:132:252-63.

112. Moriguchi Y, Decety J, Ohnishi T, Maeda M, Mori T, Nemoto K, et al. Empathy and judging other's pain: an fMRI study of alexithymia. Cereb Cortex. 2007;17:2223-34. 
113. Karlsson $H$, Näätänen $P$, Stenman $H$. Cortical activation in alexithymia as a response to emotional stimuli. Br J Psychiatry. 2008;192:32-8.

114. Moriguchi Y, Komaki G. Neuroimaging studies of alexithymia: physical, affective, and social perspectives. Biopsychosoc Med. 2013;7:8.

115. Kanbara K, Ban I, Fukunaga M, Nakai Y. The process toward awareness of bodily feeling and biofeedback [in Japanese]. Japanese J biofeedback Res. 2008:35:19-25

116. Barsky AJ, Wyshak G, Klerman GL. The somatosensory amplification scale and its relationship to hypochondriasis. J Psychiatr Res. 1990;24:323-34.

117. Barsky AJ, Brener J, Coeytaux RR, Cleary PD. Accurate awareness of heartbeat in hypochondriacal and non-hypochondriacal patients. J Psychosom Res. 1995;39:489-97.

118. Mailloux J, Brener J. Somatosensory amplification and its relationship to heartbeat detection ability. Psychosom Med. 2002;64:353-7.

119. Kano M, Fukudo S. The alexithymic brain: the neural pathways linking alexithymia to physical disorders. Biopsychosoc Med. 2013;7:1.

120. Hariri AR, Bookheimer SY, Mazziotta JC. Modulating emotional responses: effects of a neocortical network on the limbic system. Neuroreport. 2000;11:43-8.

121. Schaefer M, Egloff B, Witthöft M. Is interoceptive awareness really altered in somatoform disorders? Testing competing theories with two paradigms of heartbeat perception. J Abnorm Psychol. 2012;121:719-24.

122. Schaefer M, Egloff $B$, Gerlach AL, Witthöft M. Improving heartbeat perception in patients with medically unexplained symptoms reduces symptom distress. Biol Psychol. 2014;101:69-76.

123. Ekman P. Are there basic emotions? Psychol Rev. 1992;99:550-3.

124. Mauss IB, Levenson RW, McCarter L, Wilhelm FH, Gross JJ. The tie that binds? Coherence among emotion experience, behavior, and physiology. Emotion. 2005:5:175-90.

125. Lazarus RS. Emotion and Adaptation. New York: Oxford University Press; 1991.

126. Sze JA, Gyurak A, Yuan JW, Levenson RW. Coherence between emotional experience and physiology: Does body awareness training have an impact? Emotion. 2010;10:803-14.

127. Ikemi Y, Ikemi A. Psychosomatic medicine: a meeting ground of eastern and western medicine. J Am Soc Psychosom Dent Med. 1983;30:3.

128. Damasio AR, Everitt BJ, Bishop D. The somatic marker hypothesis and the possible functions of the prefrontal cortex. Philos Trans R Soc Lond B Biol Sci. 1996:351:1413-20.

129. Damasio AR. The body-minded brain. In: Descartes' error: emotion, reason, and the human brain. New York: Penguin; 1994. p. 223-44.

\section{Submit your next manuscript to BioMed Central and we will help you at every step:}

- We accept pre-submission inquiries

- Our selector tool helps you to find the most relevant journal

- We provide round the clock customer support

- Convenient online submission

- Thorough peer review

- Inclusion in PubMed and all major indexing services

- Maximum visibility for your research

Submit your manuscript at www biomedcentral com/submit 